\title{
VLBI Observations of Central Region in NGC 3079
}

\author{
Satoko Satoh, M. Inoue, N. Nakai, K. M. Shibata, S. Kameno, \& V. Migenes \\ National Astronomical Observatory, JAPAN
}

\author{
P. J. Diamond \\ National Radio Astronomy Observatory, Socorro, NM, U.S.A.
}

\begin{abstract}
We present multi-frequency observations towards the nucleus of NGC 3079 using both Japan VLBI Network (J-Net) and the VLBA including phased VLA and Effelsberg. We detected two continuum components at 1.4 and $8.4 \mathrm{GHz}$, and determined the spectral indices for each component. Further, we found several $\mathrm{H}_{\mathrm{l}}$ absorption features towards the continuum components whose velocity gradient is opposite to the galaxy rotation. Assuming rotation, the central mass is estimated to be $3 \times 10^{6} M_{\odot}$. The water maser distribution extends in NorthSouth direction along the galactic disk, while no clear indication of a Keplerian rotating disk was found.
\end{abstract}

\section{Introduction}

NGC 3079 has very luminous water megamaser from the nucleus, the peak of the spectrum being blueshifted by $180 \mathrm{~km} \mathrm{~s}^{-1}$ from the systemic velocity of the galaxy (Henkel et al. 1984; Haschick \& Baan 1985). Core-jet like continuum components are found in the central region within 1 pc (Irwin \& Seaquist 1988), and $\mathrm{HI}$ and $\mathrm{OH}$ absorptions were detected in the galaxy (Haschick \& Baan 1985; Irwin \& Seaquist 1991). Thus this galaxy is very unique to investigate water masers, continuum structures, and absorption features all together with VLBI.

\section{Observations and Results}

From J-Net observations at $22 \mathrm{GHz}$, we see that maser spots are spread across 4 mas in N-S direction (P.A. $\sim-10^{\circ}$ ), almost parallel to the major axis of the galaxy. VLBA observations were carried out at $1.4,8.4,15$, and $22 \mathrm{GHz}$ on December 1996. The image shows a continuum component with flux density of $14.5 \mathrm{mJy}$ located 6.7 mas west of the strongest maser at $956 \mathrm{~km} \mathrm{~s}^{-1}$ at $22 \mathrm{GHz}$. The observations give two continuum components at 1.4 and $8.4 \mathrm{GHz}$. These are aligned along NW-SE direction (P.A. $\sim-50^{\circ}$ ) within a few $\mathrm{pc}$, while only one component with FWHM $=1$ mas is detected at 15 and $22 \mathrm{GHz}$. Considering spectral continuity, the component is identified as the $\mathrm{NW}$ one in lower frequency images. The spectrum of the NW component is almost flat $\left(\alpha=0.1 ; S \propto \nu^{\alpha}\right)$, while that of the SE component is steep $(\alpha=-0.5)$. Three H I absorption features were detected towards the continuum components at the systemic velocity of the galaxy $\left(V_{\text {sys }}\right)$ and $V_{\text {sys }} \pm 100 \mathrm{~km} \mathrm{~s}^{-1}$ (Figure 1 bottom-right). Each redshifted or blueshifted feature with $\tau>0.5$ is only seen in NW or SE component, respectively, while the systemic feature is seen in both components (Figure 1). The central mass is estimated to be $3 \times 10^{6} M_{\odot}$, assuming $\mathrm{H}$ I gas is rotating at $3.4 \mathrm{mas}(1 \mathrm{pc})$ of radius and $100 \mathrm{~km} \mathrm{~s}^{-1}$ of rotation velocity. Further, it is interesting to note that at the $1 \mathrm{pc}$ scale that the direction of rotation is opposite to that of the galactic scale (Pedler et al. 1996). 


\section{Discussion}

Water masers and continuum components are significantly separated, and the elongation of these structure are different by $+40^{\circ}$. The position vs. velocity diagram of water masers along the major elongation presents no clear evidence of Keplerian rotation disk in this central region. However, absorption features suggest a systemic rotation, although the rotational center is not coincident with the NW component which could be the core by its spectrum. Disagreement of the velocity gradient of $\mathrm{H}_{\mathrm{I}}$ absorption between galactic scale and our results suggest different rotation systems of sub-pc and galactic scales, as seen in NGC 4258 (Miyoshi et al. 1995), but distribution and relation between masers and continuum structures suggest new mechanism of maser emission compared to the case of NGC 4258 .

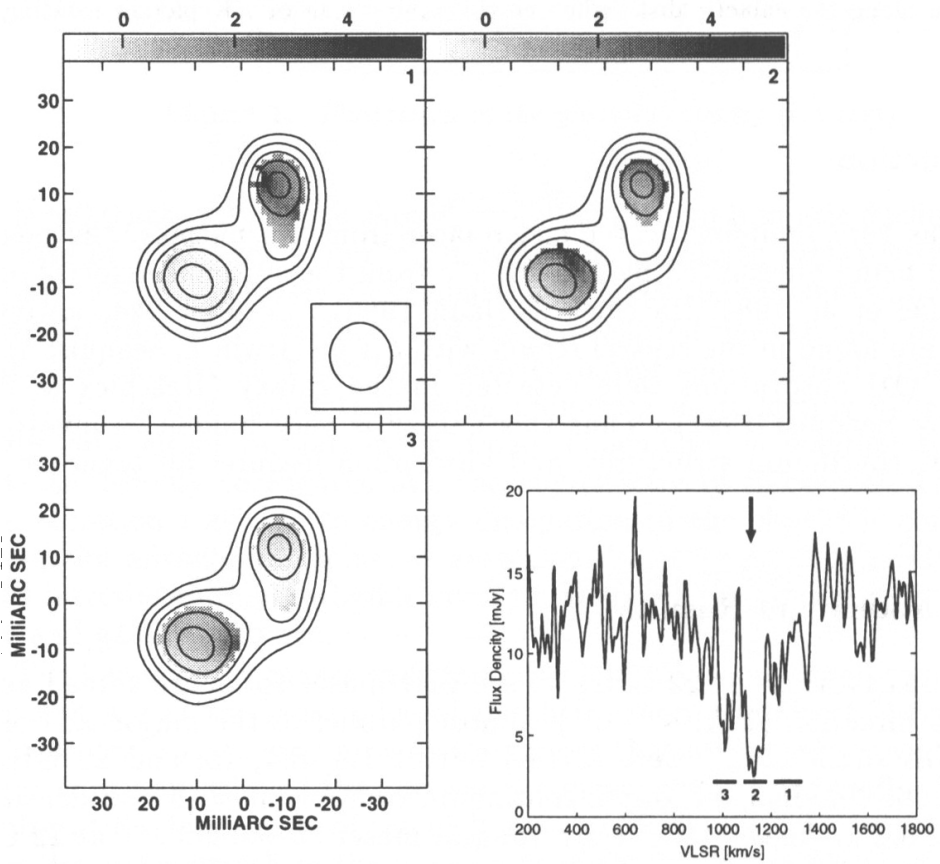

Figure 1. VLBA contour maps of continuum emission at $1.4 \mathrm{GHz}$ with grey scale images of $\mathrm{H}$ I opacity toward the nucleus. The numbers in top-right corner identify the absorption feature. (bottom-right) H I absorption features. Arrow indicates $V_{\text {sys }}$.

\section{References}

Haschick, A. D., \& Baan, W. A. 1985. Nature, 314, 144-146.

Henkel, C., et al. 1984. A\&A, 141, L1-L3.

Irwin, J. A., \& Seaquist, E. R. 1988. $A p J$, 335, 658-667.

Irwin, J. A., \& Seaquist, E. R. 1991. $A p J$, 371, 111-130.

Pedler, A., et al. 1996. Vistas in Astronomy, 40, 91-95.

Miyoshi, M., et al. 1995. Nature, 373, 127-129. 